\title{
COVID-19 - Therapiemöglichkeiten mit chinesischen Arzneimitteln in der Akutphase und Rekonvaleszenz
}

\begin{abstract}
Einleitung
Über ein halbes Jahr nach dem ersten Ausbruch der Coronapandemie ist SARS-CoV-2 weiterhin global auf dem Vormarsch und auch in den Ländern mit hoch entwickelten Gesundheitssystemen fällt es schwer, die Erkrankung unter Kontrolle zu bekommen. Die Frage der Dauer von Immunität nach überstandener Akuterkrankung oder Impfung ist noch ungeklärt, aktuell gibt es lediglich für den Wirkstoff Dexamethason unter bestimmten Voraussetzungen den Nachweis einer signifikanten Reduzierung der Mortalität bei COVID-19 (Stand Juli 2020). Außerdem gewinnt zunehmend das Problem der Folgeerkrankungen in der Rekonvaleszenzphase an Bedeutung, wie etwa Fatigue-Syndrome und persistierende restriktive Ventilationsstörungen.
\end{abstract}

\section{》) Das Problem der Folgeerkrankungen in der Rekonvaleszenzphase gewinnt an Bedeutung}

Bereits während der SARS-Epidemie 2003 wurden in China Patienten auch mit chinesischer Medizin behandelt. In einem 2004 veröffentlichten Bericht der WHO [1] wurde in einer kontrollierten Beobachtungsstudie eine signifikant höhere Überlebensrate bei Patienten festgestellt, die eine kombinierte Behandlung aus westlicher und chinesischer Medizin erhielten, verglichen mit einer ausschließlich mit westlicher Medizin behandelten Kontrollgruppe.

Auch während des aktuellen SARS-CoV-2-Ausbruchs sind im Raum Wuhan über 60.000 an COVID-19 erkrankte Patienten auch infolge einer staatlichen Anweisung - im Rahmen eines kombinierten Therapieregimes zusätzlich zu westlicher Medizin mit chinesischen Arzneimitteln (CAM) behandelt worden. In ersten Beobachtungsstudien zeigten sich für einige standardisierte CAM-Rezepturen eine signifikante Senkung von Mortalitätsraten und eine gute Therapiesicherheit [2]. Wenngleich diese Berichte noch mit einer gewissen Vorsicht zu betrachten sind und die Ergebnisse erster kontrollierter randomisierter Studien zum Einsatz von CAM bei COVID-19 noch ausstehen,

Deutsche Zeitschrift für Akupunktur 2020 • 63 (4): 208-213 https://doi.org/10.1007/s42212-020-00316-x

Online publiziert: 20. Oktober 2020

(c) Springer Medizin Verlag GmbH, ein Teil von Springer Nature 2020 kann der Einsatz chinesischer Medizin eine Bereicherung des zur Zeit noch sehr kargen Spektrums an Therapiemöglichkeiten bei SARS-CoV-2-Infektionen darstellen.

\section{I) Der Einsatz chinesischer Medizin kann eine Bereicherung darstellen}

Im vorgegebenen Rahmen dieses Zeitschriftenbeitrags können nicht Behandlungsoptionen für sämtliche Stadien der COVID19-Erkrankung in aller Ausführlichkeit diskutiert werden. Dazu sei auf weitere Quellen verwiesen [3, 4]. In der vorliegenden Arbeit sollen, basierend auf dem in China entwickelten Konsens zum dominierenden Krankheitsmechanismus und der daraufhin unter der Ägide der staatlichen Gesundheitsbehörden entwickelten Therapieprotokolle [5], Therapieoptionen für ausgewählte Krankheitsphasen einer COVID-19-Erkrankung vorgestellt werden: zum einen für die Phase der beginnenden Pneumonie, also für spätambulante bis frühstationäre Stadien, und zum anderen für mögliche Entwicklungen nach überstandener Akuterkrankung, also poststationäre Stadien mit klinischen Problemen. Dazu gehören persistierende restriktive Ventilationsstörungen oder eine Fatigue-Symptomatik, die angesichts der zunehmenden Zahl von Personen, die eine COVID-19-Erkrankung durchgemacht haben, wachsende Bedeutung erlangen und für welche die etablierte Medizin bisher keine Lösungen gefunden hat.

\section{Pathophysiologie der COVID-19-Erkrankung} aus der Sicht der chinesischen Medizin

$\mathrm{Zu}$ den Charakteristika der initialen Phase der COVID-19-Erkrankung gehört der oft milde bzw. blande Verlauf. Symptome, die aus der Sicht der chinesischen Medizin als typisch für eine Affektion der Oberfläche durch Wind-Kälte- (nach der Lehre der Erkrankungen durch Kälte - Shang Han Lun) oder WindHitze-Pathogene (Erkrankungen durch Wärme - Wen Bing) gelten, werden oft nicht beobachtet oder sind nur milde ausgeprägt. Eine Reihe der besonders oft beschriebenen Symptome wie Husten, Schwäche, Müdigkeit sowie Geruchs- und Geschmacksverlust sprechen hingegen für eine Akkumulation von trüber Nässe (Shi - Feuchtigkeit), welche die Funktionen der Funktionskreise Lunge und „Mitte“ (Magen und Milz) affizieren. Dementsprechend gibt es in der Literatur einen breiten Konsens, dass epidemisches Nässetoxisches als zentrale Pathologie von COVID-19 anzusehen ist [5-7]. 


\section{Christian Thede}

\section{COVID-19 - Therapiemöglichkeiten mit chinesischen Arzneimitteln in der Akutphase und Rekonvaleszenz}

\section{Zusammenfassung}

Angesichts der persistierenden SARS-CoV-2-Pandemie und noch immer mangelnder Therapieoptionen werden in der vorliegenden Arbeit Therapiemöglichkeiten mit chinesischen Arzneimitteln erörtert, die sich in ersten Beobachtungsstudien als Erfolg versprechend erwiesen haben. Darüber hinaus wird eine Therapieoption für die sich häufenden postinfektiösen Schwächezustände (Fatigue-Symptomatik) vorgestellt, die in ausgewählten Fällen als ein „Syndrom des kleinen Yang" betrachtet und Erfolg versprechend behandelt werden können.

Schlüsselwörter

SARS-CoV-2 $\cdot$ Pandemie $\cdot$ Fatigue-Syndrom $\cdot$ TCM

\section{COVID-19—therapeutic options using Chinese herbal medicines in the acute phase and convalescence}

\section{Abstract}

In light of the persistent SARS-CoV-2 pandemic and the current lack of treatment options, this work discusses therapeutic options using Chinese herbal medicines that have shown promise in initial observational studies. In addition, a therapeutic option for the increasing number of postinfectious states of weakness (fatigue symptoms) is presented, which in selected cases can be viewed as a "lesser yang syndrome" and treated with promise.

Keywords

SARS-CoV-2 - Pandemic · Fatigue-Syndrome $\cdot$ TCM
Auch im Stadium der COVID-19-Pneumonie wird das Krankheitsgeschehen von einer Blockade der Funktionen der Funktionskreise Lunge und „Mitte“ dominiert, bedingt durch die Akkumulation von Nässe (Shi) im Bereich des oberen und mittleren Erwärmers (Sanjiao). In diesem Stadium kann die Erkrankung einen unterschiedlichen Verlauf nehmen:

- Da in der Phase des ersten Ausbruchs in der Region Wuhan im Januar noch winterliche Temperaturen herrschten, sind aus dieser Zeit in der genannten Region vorwiegend Verläufe mit Zeichen der Blockade des pulmonalen Qi wie Husten und Dyspnoe, Symptomen der Nässeaffektion der Funktionskreise Magen und Milz wie Appetitlosigkeit, Übelkeit, Diarrhö, Schwäche, Müdigkeit sowie mäßiges Fieber ohne Wärmeintoleranz beschrieben worden. Die Zungen der Betroffenen waren in der Regel blass und gedunsen mit weißem feuchten Belag.

- Während des Fortschreitens der Epidemie innerhalb Chinas im Verlauf des einsetzenden Frühlings sind bei wärmeren Außentemperaturen aus anderen Provinzen Chinas auch Symptome und Zeichen berichtet worden, die auf die Entwicklung einer Nässe-Hitze-Pathologie hinweisen, wie z. B. ausgeprägteres Fieber mit Wärmeintoleranz, erschwerte Defäkation, gelb gefärbtes Sputum sowie gerötete Zungen mit gelblichem Belag. Obgleich beiden Verlaufsformen als zentrale Pathologie die Akkumulation von Nässe gemeinsam ist, ist es nach allgemeinem Konsens der Experten von großer Bedeutung, den Temperaturaspekt der Erkrankung korrekt zu beurteilen und bei der Verschreibung von chinesischen Arzneimitteln zu berücksichtigen.

Sollte die Erkrankung von den beschriebenen Anfangsstadien der Pneumonie weiter fortschreiten, kann es zu verschiedenen Komplikationen kommen, die aus der Sicht der chinesischen Medizin folgendermaßen charakterisiert werden:

- Hitze und Stagnation von Toxischem blockieren den Funktionskreis Lunge
Dieses Stadium entspricht der Phase der diffusen interstitiellen Pneumonie mit überschießender Immunreaktion („Zytokinsturm") und alveolärer Überladung, gekennzeichnet $\mathrm{u}$. a. durch Dyspnoe, beschleunigte Atmung, hohes Fieber, evtl. Affektion weiterer Organe.

- Hitze und Stagnation führen zu Blutstase

Typisch hierfür sind Gefäßkomplikationen, wie z. B. thromboembolische Prozesse in verschiedenen Regionen und Organen. Hier scheinen als Risikopersonen besonders Infizierte betroffen zu sein, die bereits unter Vorerkrankungen aus dem Formenkreis des metabolischen Syndroms und der arteriellen Verschlusskrankheit leiden - Erkrankungen, die aus der Sicht der chinesischen Medizin oft mit einer Tendenz zur Blutstase assoziiert sind.

- Weitere in China vorgenommene Differenzierungen eines sich lebensbedrohlich fortentwickelnden Krankheitsbildes wie: Hitze führt zur Blockade im Inneren, zum Verbrennen des Yin und Kollaps des Yang, beschreiben klinische Zustände, die wir unter intensivmedizinischen Bedingungen bei Multiorganversagen, septischen Zuständen und Beatmungspflichtigkeit beobachten.

In der Phase der Rekonvaleszenz werden sowohl bei Infizierten nach Überstehen einer schweren COVID-19-Pneumonie als auch bei Personen, die eine milde oder komplett symptomfreie Akutinfektion durchgemacht haben, persistierende Probleme wie restriktive Ventilationsstörungen auch nach Abklingen der akuten Entzündungsprozesse beobachtet. Für diese Folgeerkrankungen werden aus der Sicht der chinesischen Medizin Störungsmuster wie Qi- und Yin-Defizit sowie persistierende Schleim-(Tan-)Befunde verantwortlich gemacht und entsprechende Therapiekonzepte empfohlen [5].

In zunehmendem Maße leiden außerdem ehemals SARSCoV-2-Infizierte unter einer Fatigue-Symptomatik, die den Beschwerden nach Epstein-Barr-Virus-(EBV-)Infektionen ähnelt [8]. Während coronavirusassoziierte Fatigue-Symptomatik bis- 
her in der chinesischen Literatur keinen Niederschlag findet, können die Beschwerden nach eigenen Erfahrungen des Autors zumindest teilweise als „Syndrom des Kleinen Yang“ (ShaoyangSyndrom) im Sinne der „Abhandlung über Erkrankungen durch Kälte" (Shang Han Lun) interpretiert und behandelt werden, was in der vorliegenden Arbeit vorgestellt werden soll.

\section{Therapiekonzepte mit chinesischen Arzneimitteln für ausgewählte Krankheitsstadien von COVID- 19-Erkrankungen}

Die in chinesischen Beobachtungsstudien berichteten Behandlungserfolge beziehen sich vorwiegend auf die Rezeptur „den Funktionskreis Lunge kühlendes und Toxisches ausscheidendes Dekokt“ (Qingfei Paidu Tang), einer Rezeptur, die aus 4 klassischen Arzneimittelrezepturen zusammengesetzt ist und über 20 Einzelarzneien beinhaltet $[2,5]$. In den staatlichen Therapieprotokollen sind allerdings auch zahlreiche differenzierte Therapieempfehlungen für unterschiedliche Krankheitsstadien und Verlaufsformen der Erkrankung aufgeführt.

Basierend auf diesen Angaben werden im Folgenden Behandlungskonzepte für folgende ausgewählte klinische Stadien vorgestellt:

1. frühe und mittlere Stadien der COVID-19-Pneumonie,

2. respiratorische Probleme und Fatigue-Symptomatik in der Rekonvaleszenz nach Überstehen der Akutinfektion.

\section{Frühe und mittlere Stadien der COVID-19-Pneumonie}

Bei Erkrankten mit beginnender Lungenbeteiligung besteht das Therapieziel darin, die Ausprägung interstitieller Infiltrate mit massiven alveolären Exsudationen zu vermeiden bzw. abzumildern, um der Entwicklung einer terminalen beatmungspflichtigen respiratorischen Insuffizienz entgegenzuwirken. Das Anfangsstadium der sich entwickelnden Pneumonie - für die Betroffenen noch in der ambulanten oder bereits in der frühstationären Behandlungsphase - ist in der Regel die letzte realisierbare Möglichkeit, den Erkrankungsverlauf mithilfe der chinesischen Medizin noch positiv beeinflussen zu können.

Abhängig von der Zahl von Erkrankten und Belastung der Kliniken dürfte der Zeitpunkt der Aufnahme in klinisch-stationäre Behandlung variieren. Teilweise werden evtl. auch Erkrankte mit bereits manifester Pneumonie ambulant zu behandeln sein, sodass unter Umständen auch Therapeuten, die nicht im klinisch-stationären Bereich tätig sind, die Möglichkeit haben, in dieser Zeit einer gravierenden Verschlechterung des Allgemeinzustands entgegenzuwirken, indem durch frühzeitige Elimination der Akkumulation von Nässe (Shi) der Entstehung von manifester Stagnation von Toxischem mit Hitze $(R e)$ entgegengewirkt wird, um somit der kritischen Reduzierung der alveolären Funktion mit konsekutiver Notwendigkeit einer maschinellen Beatmung vorzubeugen. Angesichts der nach wie vor reduzierten Prognose beatmeter COVID-19-Patienten ist die beschriebene frühzeitige Therapie bei noch mild ausgeprägter Symptomatik von besonderem Wert.

Nach den Prinzipien der chinesischen Medizin sollten therapeutische Konzepte auch für COVID-19-Patienten an den erkennbaren Zeichen bzw. Symptomen und somit dem jeweiligen Stadium der Erkrankung orientiert sein. Nach dem Muster einer universalspezifischen Wirksamkeit zusammengestellte Rezepte mindern die Erfolgschancen der Therapie und vergrößern
Tab. 1 Rezeptur COVID-19 A

\begin{tabular}{l|l}
\hline Ephedrae herba (Mahuang) & $6 \mathrm{~g}$ \\
\hline Armeniacae semen amarum (Kuxingren) & $9 \mathrm{~g}$ \\
\hline Agastachis herba (Huoxiang) & $9 \mathrm{~g}$ \\
\hline Magnoliae cortex (Houpo) & $9 \mathrm{~g}$ \\
\hline Atractylodis rhizoma (Cangzhu) & $9 \mathrm{~g}$ \\
\hline Lepidii/Descurainiae semen (Tinglizi) & $9 \mathrm{~g}$ \\
\hline Platycodi radix (Jiegeng) & $9 \mathrm{~g}$ \\
\hline Pinelliae rhizoma praep. (Zhibanxia) & $9 \mathrm{~g}$ \\
\hline Citri reticulatae pericarpium (Chenpi) & $6 \mathrm{~g}$ \\
\hline Poria (Fuling) & $9 \mathrm{~g}$ \\
\hline Arecae pericarpium (Dafupi) & $6 \mathrm{~g}$ \\
\hline Sinapis albae semen (Baijiezi) & $9 \mathrm{~g}$ \\
\hline Zingiberis rhizoma recens (Shengjiang) & $6 \mathrm{~g}$ \\
\hline Jujubae fructus (Dazao) & $3 \mathrm{~g}$
\end{tabular}

das Potenzial unerwünschter Nebenwirkungen. Die vorgestellten Konzepte basieren auf dem aktuellen Konsens in der Literatur und sind als Vorschläge zur Therapie zu betrachten, die der jeweiligen klinischen Situation anzupassen sind. Die angegebenen Rezepturen zielen auf ambulante Stadien mit bereits begonnener Lungenbeteiligung bis hin zu klinisch behandlungsbedürftigen Phasen, in denen keine bzw. noch keine invasive Beatmung notwendig ist.

Auf der Basis der in den chinesischen Quellen angegebenen zahlreichen Rezepturempfehlungen wurden vom Autor dieses Artikels Basisrezepturen für 3 wichtige Grundkonstellationen pneumonischer Stadien der COVID-19-Erkrankung entwickelt, die hier mit den jeweils wichtigsten Modifikationen für die Anpassung der Arzneimittelzusammensetzung an individuelle Erfordernisse vorgestellt werden:

\section{Akkumulation von Schleim-Nässe (Tanshi) behindert Entfaltung des Qi des Funktionskreises Lunge}

Frühe COVID-19-Stadien mit Lungenbeteiligung ohne klinische Zeichen von Hitze $(R e)$.

\section{Symptome}

Husten ohne oder mit wenig zähem farblosen Schleim, leichte Atemnot, thorakales Druckgefühl, mäßiges Fieber ohne Hitzeempfinden, evtl. Frösteln, Schwäche, Müdigkeit, evtl. Unwohlsein, Appetitlosigkeit, Durchfall, Geschmacks- und Geruchsverlust. Zunge: Körper blass, gedunsen, Belag feucht und weiß bzw. grau und schmierig. Pulse: schlüpfrig (Hua).

\section{Therapieprinzipien}

Umwandlung und Kanalisierung von Schleim (Tan) und Nässe (Shi), Entfaltung des Qi des Funktionskreises Lunge sowie Stützung und Harmonisierung der „Mitte“.

\section{Therapie}

Rezeptur COVID-19 A (Tab. 1) zur Elimination von Nässe (Shi) und Entfaltung des Qi des Funktionskreises Lunge. 


\section{Tab. 2 Rezeptur COVID-19 B}

\begin{tabular}{|l|l|}
\hline Ephedrae herba (Mahuang) & $6 \mathrm{~g}$ \\
\hline Armeniacae semen amarum (Kuxingren) & $9 \mathrm{~g}$ \\
\hline Coicis semen (Yiyiren) & $9 \mathrm{~g}$ \\
\hline Magnoliae cortex (Houpo) & $9 \mathrm{~g}$ \\
\hline Pinelliae rhizoma praep. (Zhibanxia) & $9 \mathrm{~g}$ \\
\hline Benincasae semen (Dongguazi) & $9 \mathrm{~g}$ \\
\hline Scutellariae radix (Huangqin) & $9 \mathrm{~g}$ \\
\hline Poria (Fuling) & $9 \mathrm{~g}$ \\
\hline Lepidii/Descurainiae semen (Tinglizi) & $9 \mathrm{~g}$ \\
\hline Amomi fructus rotundus (Baidoukou) & $9 \mathrm{~g}$ \\
\hline Glycyrrhizae radix (Gancao) & $6 \mathrm{~g}$ \\
\hline
\end{tabular}

Das Rezept ist eine modifizierte Kombination folgender Rezepturen: „Pulver mit Agastache zur Stärkung des geradläufigen Qi“ (Huoxiang Zhengqi San), „Ephedra-Dekokt“" (Mahuang Tang), „Dekokt mit Lepidium und Jujuba zur Zerstreuung des Funktionskreises Lunge" (Tingli Dazao Xiefei Tang).

Hervorzuhebende Modifikationen (ausführliche Modifikationen: $[3,4])$

- Bei Symptomatik des kleinen Yang (Shaoyang) wie bitterer Mundgeschmack, Wechsel von Frösteln und Wärme: +Bupleuri radix (Chaihu), Scutellariae radix (Huangqin)

- Bei Anzeichen für Blutstase: +Carthami flos (Honghua), Chuanxiong rhizoma (Chuanxiong), Paeoniae radix rubra (Chi Shao), Angelicae sinensis radix (Danggui), Salviae miltiorrhizae radix (Danshen), Curcumae radix (Yujin)

\section{Nässe-Hitze (Shire) affiziert den Funktionskreis Lunge}

Frühe und mittlere Stadien der COVID-19-Pneumonie mit Nässe (Shi) und Zeichen von Hitze (Re).

\section{Symptome}

Husten ohne oder mit wenig zähem gelblichen Schleim, leichte Atemnot, thorakales Druckgefühl, mäßiges Fieber, Schwäche, Schweregefühl, evtl. Unwohlsein, Appetitlosigkeit, Defäkation erschwert bei weichem Stuhl. Zunge: blass oder gerötet, Belag gelblich und schmierig. Pulse: schlüpfrig (Hua), evtl. beschleunigt $(S h u)$.

\section{Therapieprinzip}

Umwandlung und Kanalisierung von Nässe (Shi), Ausleitung von Nässe-Hitze (Shire) sowie der Stagnation von Toxischem, Entfaltung des Qi des Funktionskreises Lunge.

\section{Therapie}

Rezeptur COVID-19 B (Tab. 2) zur Umwandlung von Nässe (Shi), zur Ausleitung von Nässehitze (Shire) und zur Lösung von Blockaden des Funktionskreises Lunge.

Das Rezept ist eine modifizierte Kombination folgender Rezepturen: „Dekokt mit Ephedra, Prunus armeniaca, Coicis und Glycyrrhiza“ (Maxing Yigan Tang), „Dekokt der drei Samen“ (Sanren Tang).

Hervorzuhebende Modifikationen (ausführliche Modifikationen: $[3,4])$
Tab. 3 Rezeptur COVID-19C

\begin{tabular}{|l|l|}
\hline Ephedrae herba (Mahuang) & $6 \mathrm{~g}$ \\
\hline Armeniacae semen amarum (Kuxingren) & $9 \mathrm{~g}$ \\
\hline Gypsum fibrosum (Shigao) & $30 \mathrm{~g}$ \\
\hline Glycyrrhizae radix (Gancao) & $3 \mathrm{~g}$ \\
\hline Lepidii/Descurainiae semen (Tinglizi) & $10 \mathrm{~g}$ \\
\hline Platycodi radix (Jiegeng) & $10 \mathrm{~g}$ \\
\hline Trichosanthis semen (Gualouren) & $9 \mathrm{~g}$ \\
\hline Agastachis herba (Huoxiang) & $10 \mathrm{~g}$ \\
\hline Magnoliae cortex (Houpo) & $10 \mathrm{~g}$ \\
\hline Poria (Fuling) & $15 \mathrm{~g}$ \\
\hline Lonicerae flos (Jinyinhua) & $15 \mathrm{~g}$ \\
\hline Forsythiae fructus (Lianqiao) & $15 \mathrm{~g}$ \\
\hline Houttuyniae herba (Yuxingcao) & $30 \mathrm{~g}$ \\
\hline Persicae semen (Taoren) & $9 \mathrm{~g}$ \\
\hline Rhei rhizoma (Dahuang) & $6 \mathrm{~g}$ \\
\hline
\end{tabular}

- Einstau von Hitze mit Obstipation: +Gypsum fibrosum (Shigao), Persicae semen (Taoren), Achyranthis bidentatae radix (Niuxi) bzw. + „Dekokt des weißen Tigers“ (Baihu Tang)

- Hohes Fieber, reduzierter Allgemeinzustand: +Houttuyniae herba (Yuxingcao), Lonicerae flos (Jinyinhua), Forsythiae fructus (Lianqiao)

- Anzeichen für Blutstase: siehe Modifikation COVID-19 A

\section{Hitze $(R e)$ und Stagnation von Toxischem blockieren den Funktionskreis Lunge}

Fortgeschrittenes Krankheitsstadium bei COVID-19 mit zunehmender Atemnot, erschwerter und beschleunigter Atmung.

\section{Symptome}

Schwere Atemnot, beschleunigte flache Atmung, Cyanose, Husten ohne oder mit wenig zähem gelblichen Schleim, Druck- bzw. Engegefühl, Fieber, Hitzegefühl, Schwäche, Schweregefühl, stark reduzierter Allgemeinzustand, evtl. Unwohlsein, Appetitlosigkeit, Obstipation, dunkler, spärlicher Urin. Zunge: stark gerötet, evtl. livide, Belag trocken, dick, gelb bzw. gelbbraun. Pulse: schlüpfrig $(H u a)$, evtl. beschleunigt $(\mathrm{Shu})$.

\section{Therapieprinzip}

Erweichung und Umwandlung von Schleim (Tan), Umwandlung und Kanalisierung von Nässe (Shi) bzw. Schleim (Tan), Klärung und Ableitung von Hitze $(R e)$ und Toxischem, Entfaltung des Funktionskreises Lunge.

\section{Therapie}

Rezeptur COVID-19 C (Tab. 3) zum Eliminieren von Hitze (Re), Nässe (Shi) und Stagnation von Toxischem zur Entfaltung des o. Funktionskreises Lunge.

Das Rezept ist eine modifizierte Kombination folgender Rezepturen: „Dekokt mit Ephedra, Prunus armeniaca, Gypsum und Glycyrrhiza“ (Maxing Shigan Tang), „große Pille für Gebundenes in der Brust" (Da Xian Xiong Wan). 
Hervorzuhebende Modifikationen (ausführliche Modifikationen: $[3,4])$

- Trockenheit, zäher Schleim: +Phragmitis rhizoma (Lugen), Trichosanthis radix (Tianhuafen), Fritillariae cirrhosae bulbus (Chuanbeimu)

- Zum Ableiten von Hitze $(R e)$ : +Achyranthis bidentatae radix (Niuxi), Natrii sulfas (Mangxiao)

- Anzeichen für Blutstase: siehe Modifikation COVID-19 A

Anmerkungen zur Arzneimittelsicherheit der vorgestellten Rezepturen:

- Die genannten Rezepturen sind aufgrund ihrer Bestandteile in der Schwangerschaft kontraindiziert.

- Bei der Anwendung von Ephedrae herba (Mahuang) sind Aspekte der Arzneimittelsicherheit zu bedenken. Es müssen die Kontraindikationen, Wechselwirkungen und möglichen Interaktionen mit „westlichen“ Medikamenten im Sinne der Vorgaben der Kommission E berücksichtigt werden. Letztlich muss die Anwendung der Arznei im Rahmen einer Abwägung der Nutzen und Risiken angesichts des potenziell schweren Krankheitsverlaufs in Relation zu den Einnahmerisiken erfolgen. In den angegebenen Dosierungen und im Kontext der verwendeten Rezepturen gilt hier Ephedrae herba (Mahuang) als eine der zentralen Arzneien zur Lösung der Blockade des pulmonalen Qi. Ein Ersatz durch andere Drogen wird dort nicht empfohlen ${ }^{1}[10]$.

\section{Rekonvaleszenzphase}

Dominierende Probleme in der Zeit nach überstandener Akutinfektion sind persistierende respiratorische Beschwerden sowie Schwächezustände.

1. Respiratorische Symptomatik ist aus der Sicht der chinesischen Medizin hauptsächlich bedingt durch ein Qi- bzw. Yin-Defizit des Funktionskreises Lunge sowie durch Persistenz von Trübem, sodass Schleim (Tan) das pulmonale Qi nicht zur Entfaltung kommen lässt. Im Fall von $Q i$-Defizit, oft gekennzeichnet durch Dyspnoe mit Ermüdbarkeit und spontanen Schweißausbrüchen, wird oft das „Dekokt zur Stützung des Funktionskreises Lunge“ (Bufei Tang) empfohlen. Alternativ oder begleitend können Zeichen von Trockenheit bei Yin-Defizit auftreten. Für diese Problematik werden vielfach Rezepturen wie „Liliendekokt zur Festigung des Metalls“ (Baihe Gujin Tang) oder „Dekokt mit Glehnia und Ophiopogonis“ (Shashen Maimendong Tang) verwendet. Eine etablierte Rezeptur für eingetrockneten Schleim (Tan) ist das „Pulver mit Fritillaria und Trichosanthis“ (Beimu Gualou San). Allerdings ist beim derzeitigen Wissensstand noch unklar, in welchen Fällen respiratorische Probleme nach SARS-CoV-2-Infektionen überhaupt reversibel sind. Leider gibt es zunehmend Hinweise darauf, dass sich bei einer Reihe von Betroffenen auch nach überstandener Infektion parenchymatöse Umbauprozesse im Sinne einer Lungenfibrose einstellen, die zumindest bei einem Teil der Patienten zu irreversiblen und therapieresistenten restriktiven Ventilationsstörungen führen könnten [9].

2. Immer häufiger wird über Zustände von Schwäche und Ermüdbarkeit berichtet, die an Fatigue-Syndrome, wie z. B. nach EBV-Infektionen, erinnern [8]. Etablierte Therapieempfeh-

1 Prof. Zhijun Jie, Director of Department of Pulmonary and Critical Care Medicines, The Fifth People's Hospital of Shanghai, Fudan University - persönliche Mitteilung.
Tab. 4 Modifikation des kleinen Bupleurum Dekokts

\begin{tabular}{l|l}
\hline Bupleuri radix (Chaihu) & $9 \mathrm{~g}$ \\
\hline Scutellariae radix (Huangqin) & $6 \mathrm{~g}$ \\
\hline Pinelliae rhizoma praep. (Zhibanxia) & $9 \mathrm{~g}$ \\
\hline Codonopsis radix (Dangshen) & $6 \mathrm{~g}$ \\
\hline Glycyrrhizae radix (Gancao) & $3 \mathrm{~g}$ \\
\hline Zingiberis rhizoma recens (Shengjiang) & $6 \mathrm{~g}$ \\
\hline Jujubae fructus (Dazao) & $3 \mathrm{~g}$ \\
\hline Platycodi radix (Jiegeng) & $6 \mathrm{~g}$ \\
\hline Trichosanthis fructus (Gualou) & $6 \mathrm{~g}$ \\
\hline Fritillariae cirrhosae bulbus (Chuanbeimu) & $6 \mathrm{~g}$
\end{tabular}

lungen für diese Problematik aus China liegen aktuell noch nicht vor, doch soll an dieser Stelle aufgrund eigener Erfahrungen des Autors darauf hingewiesen werden, dass es für versierte Therapeuten der chinesischen Arzneimitteltherapie Erfolg versprechend sein kann, nach Anzeichen eines Syndroms des kleinen Yang (Shaoyang-Syndrom nach der „Abhandlung über Erkrankungen durch Kälte") zu forschen. In diesen Fällen kann die Verordnung des „kleinen Bupleurum-Dekokts“ (Xiao Chaihu Tang) bzw. Modifikationen der Rezeptur zur raschen, kompletten und dauerhaften Besserung führen.

\section{Fallbeschreibung}

Ein 44-jähriger Mann ohne nennenswerte Vorerkrankungen klagte nach einer vor 2 Monaten durchgemachten SARS-CoV2-Infektion mit milder Atemwegssymptomatik (Husten, Dyspnoe, Fieber - ambulante Therapie in häuslicher Quarantäne) über Schwächezustände (mit fortgesetzter Arbeitsunfähigkeit), extrem verschlimmert durch leichte Belastungen, wie z. B. Treppensteigen über eine Etage, und kombiniert mit Schweißausbrüchen, Benommenheit, Husten mit spärlichem zähen Sputum, thorakalem Druckgefühl, ständigem Unwohlsein, Wechsel von Kälte- und Wärmeempfindlichkeit.

Unter der Diagnose eines postinfektiösen Shaoyang-Syndroms wurde folgende Modifikation des kleinen Bupleurum Dekokts verordnet (Tab. 4):

Nach kurzer Einnahme reagierte der Patient mit verstärktem Husten mit vermehrtem Sputum. Daraufhin wurden Belamcandae rhizoma (Shegan) $6 \mathrm{~g}$ und Magnoliae cortex (Houpo) $6 \mathrm{~g}$ ergänzt.

Nach insgesamt vierwöchiger Einnahme der Verschreibung konnte die Behandlung wegen Beschwerdefreiheit beendet werden.

\section{Literatur}

1. World Health Organization (2004) SARS —Clinical trials on treatment using a combination of Traditional Chinese medicine and Western medicine. World Health Organization, Geneva (Report of the WHO International Expert Meeting to review and analyse clinical reports on combination treatment for SARS, 8-10 October 2003, Beijing, People's Republic of China)

2. Yang Y, Islam MS, Wang J, Li Y, Chen X (2020) Traditional Chinese Medicine in the treatment of patients infected with 2019-New Coronavirus (SARS-CoV-2): a review and perspective. Int J Biol Sci 16(10):1708-1717 
3. Thede C (2020) Mögliche Therapiestrategien bei Covid-19-Erkrankungen mit chinesischen Arzneimitteln. Chin Med 35(2):55-78

4. Thede C, Nögel R, Simon A COVID-19-Strategien zur Prävention und Therapie mit Methoden der Chinesischen Medizin. www.tcm.edu/tcm-covid-19/chinesischearzneimitteltherapie/. Zugegriffen: 06.2020

5. National Health Commission of the PRC, National Administration of Traditional Chinese Medicine of the PRC (2020) Guidance for Corona Virus Disease 2019_prevention, control, diagnosis and management. People's Medical Publishing House, Beijing

6. Xue Y, Zhang W, Xu GH et al (2020) The dampness epidemic: exploring the clinical characteristics of COVID-19 in Shanghai. Shanghai J Tcm Pharmacol: (Redacted and translated by Heiner Fruehauf. www.classicalchinesemedicine.org. Zugegriffen: 06/2020)

7. Zhao J, Flanagan C (2020) Approaching covid-19 as damp toxin. Lantern 172a:16-23

8. Carfi A, Bernabei R, Landi F, Gemelli Against COVID-19 Post-Acute Care Study Group, Gemelli Against COVID-19 Post-Acute Care Study Group (2020) Persistent symptoms in patients after acute COVID-19. JAMA. https://doi.org/10.1001/ jama.2020.12603

9. Grillo F, Barisione E, Ball L et al (2020) Lung fibrosis: an undervalued finding in COVID-19 pathological series. Lancet Infect Dis. https://doi.org/10.1016/S14733099(20)30582-X

10. Chen J, Huang D, Wang SQC Medical records from a young and brave female traditional Chinese medicine (TCM) doctor on fighting the COVID-19. www.elotus. org. Zugegriffen: 06.2020 (Zusammengestellt und ins Englische übersetzt von Chen JK)

\section{Korrespondenzadresse}

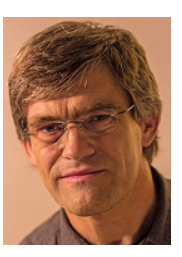

\section{Dr. Christian Thede}

Gorch-Fock-Weg 16, 23730 Neustadt, Deutschland Info@praxis-thede.de

\section{Einhaltung ethischer Richtlinien}

Interessenkonflikt. C. Thede gibt an, dass kein Interessenkonflikt besteht.

Für diesen Beitrag wurden vom Autor keine Studien an Menschen oder Tieren durchgeführt. Für die aufgeführten Studien gelten die jeweils dort angegebenen ethischen Richtlinien.
Fuir Autoren

\section{Möchten Sie einen Beitrag für die Deutsche Zeitschrift für Akupunktur einreichen?}

Wir freuen uns, dass Sie die DZA mitgestalten möchten.

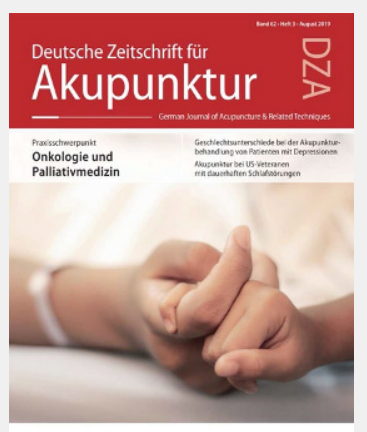

פ̦ Springer Medizin

Für folgende Rubriken können Manuskripte eingereicht werden:

- Review

- Originalien

Um Ihnen bei der Manuskripterstellung behilflich zu sein, haben wir für unsere Autoren ausführliche Autorenleitfäden und Musterbeiträge für die verschiedenen Rubriken zusammengestellt.

Diese und weitere Hinweise zur Manuskripterstellung finden Sie online unter dem Menüpunkt "Hinweise für Autoren" unter www.springer.com/journal/42212

Bitte reichen sie Ihre Manuskripte über den Editorial Manager ein. Wählen Sie hierzu auf der Zeitschriftenhomepage www.springer.com/journal/42212 den Navigationspunkt "For authors" und anschließend "Submit manuscript".

Bei Fragen zur Einreichung wenden Sie sich bitte an den zuständigen Rubrikherausgeber Herrn PD Dr. Johannes Fleckenstein: johannes.fleckenstein@sport.uni-frankfurt.de

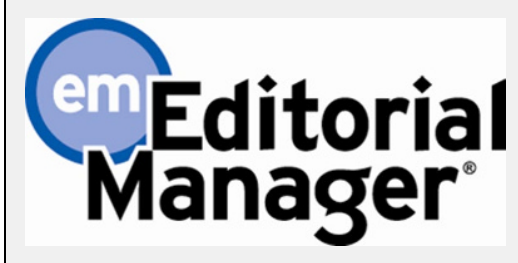

Wir freuen uns auf Ihre Beiträge!

Ihre Redaktion der DZA 